\section{Combined use of covered and uncovered self-expandable metal stents in patients with bleeding, obstructing stage IV colorectal cancer}

Endoscopic placement of self-expandable metal stents (SEMS) represents a valid therapeutic method in selected patients with colorectal obstruction [1-5]. Here we report on a series of three patients with bleeding, obstructing stage IV colorectal cancer (CRC), who were initially treated with a covered stent, which was later replaced with an uncovered one. Uncovered stents rarely dislodge [5], reducing the possibility of acute colorectal obstruction after patient discharge.

Three patients (mean age 72 years; two men, one woman) were admitted to the hospital with major rectal bleeding. At endoscopy, a CRC was identified, with ulcerated, bleeding mucosa. The cancer was located in the sigmoid colon in two patients and in the upper rectum in one patient. In each case, the lumen was almost completely obstructed by the tumor. A computed tomography scan was performed and showed diffuse liver metastasis that was considered to be unresectable. Multiple bleeding sites were controlled using argon laser but with difficulty. At the end of the procedure there was still some bleeding from the tumor. A covered SEMS (TaeWoong TTS full covered colonic stent; $10 \mathrm{~cm}$ in length, $28 \mathrm{~mm}$ in diameter; TaeWoongMedical Co., Ltd., Gimpo-si, Gyeonggi-do, South Korea) was placed un- der endoscopic and fluoroscopic control in order to apply radial pressure to the bleeding sites. At the end of the procedure there was no evidence of bleeding.

At 10 days after the procedure, an endoscopy was performed and the covered stent was removed. There was no evidence of bleeding. A 9-cm uncovered stent $(9 \mathrm{~cm}$ in length, $25 \mathrm{~mm}$ in diameter; Boston Scientific, Natick, Massachusetts, USA) was placed. At a mean of 10 months after the procedure, none of the patients complained of bleeding or of symptoms of recurrent obstruction.

The cases described here presented an unusual scenario of patients with stage IV CRC (with diffuse liver metastases), with an obstructing, bleeding cancer, for which argon laser treatment was only partially successful: there was still bleeding from the tumor surface at the end of the procedure. We decided to place a covered SEMS in order to apply radial pressure to the bleeding tumor and stop the residual bleeding. The procedure was successful in all three patients, both in controlling the bleeding and relieving the obstruction.

Endoscopy_UCTN_Code_TTT_1AS_2AZ

Competing interests: None
Antonietta Lamazza, Enrico Fiori, Antonio V. Sterpetti

Istituto Pietro Valdoni, University of Rome La Sapienza, Rome, Italy

\section{References}

1 Lee JH, Ross WA, Davila R et al. Self-expandable metal stents (SEMS) can serve as a bridge to surgery or as definitive therapy in patients with an advanced stage of cancer: clinical experience of a tertiary cancer center. Dig Dis Sci 2010; 55: 3530-3536

2 Baron TH. Colonic stenting: a palliative measure only or a bridge to surgery? Endoscopy 2010; 42: $163-168$

3 Lamazza A, DeMasi E, Fiori $E$ et al. Self expanding metal stents for treatment of anastomotic complication after colorectal resection. Endoscopy 2013; 45: 493 - 495

4 Lamazza A, Fiori E, Schillaci A et al. A new technique for self expandable metal stent placement in patients with colorectal obstruction. Surg Endosc 2013; 27: 1045 1048

5 Lamazza A, Fiori E, Schillaci A et al. Self expandable metal stents in patients with stage IV unresectable colorectal cancer. World J Surg 2012; 36: 2931-2936

\section{Bibliography}

DOI http://dx.doi.org/

10.1055/s-0033-1359187

Endoscopy 2014; 46: E244

(c) Georg Thieme Verlag KG

Stuttgart · New York

ISSN 0013-726X

Corresponding author

Antonietta Lamazza and Antonio V. Sterpetti

Department of Surgery

University of Rome

Policlinico Umberto I

Viale del Policlinico 155

00167 Rome

Italy

Fax: +39-6-49972215

antonietta.lamazza@uniroma1.it

antonio.sterpetti@uniroma1.it 